Please quote as: Wegener, R.; Prinz, A. \& Leimeister, J. M. (2013): Mobiles Lernen mit Tablet PCs in universitären Massenveranstaltungen - Eine empirische Untersuchung von Einflussfaktoren auf Nutzungsintention und Lernendenzufriedenheit. Mobile Learning: Potenziale, Einsatzszenarien und Perspektiven des Lernens mit mobilen Endgeräten. Aufl./Vol.. Hrsg./Editors: de Witt, C. \& Sieber, A. Verlag/Publisher: Springer, Wiesbaden, Germany. Erscheinungsjahr/Year: 2013. Seiten/Pages: 101-120 (DOI: 10.1007/978-3-53119484-4). 


\title{
6 Mobiles Lernen mit Tablet PCs in universitären Massenveranstaltungen - Eine empirische Untersuchung von Einflussfaktoren auf Nutzungsintention und Lernendenzufriedenheit
}

\author{
René Wegener/Andreas Prinz/Jan Marco Leimeister
}

Abstract: Mobile Learning bietet das Potenzial, nicht nur ein rein selbstgesteuertes E-Learning immer und überall zu ermöglichen. Vielmehr erlaubt es, E-LearningAnwendungen auch innerhalb von Vorlesungssälen einzusetzen. Hierbei sind jedoch grundlegend andere Einflüsse auf die Nutzungsintention und letztliche Nutzung durch die Lernenden vorhanden als in selbstgesteuerten Lernszenarien. Bisherige Studien adressieren nur unzureichend, inwiefern sich selbstgesteuertes und präsenzgebundenes Mobile Learning unterscheiden. Um diese Forschungslücke zu schließen, wurde eine Studie im Rahmen einer Massenveranstaltung an der Universität Kassel durchgeführt. Dabei wurden Mobile Learning-Anwendungen sowohl innerhalb als auch außerhalb des Vorlesungssaals eingesetzt und evaluiert. Auf Basis des UTAUT-Modells wurden mittels Online-Befragung die Einflussfaktoren auf die Nutzungsintention von präsenzgebundenem und selbstgesteuertem Mobile Learning mittels Partial Least Squares (PLS) berechnet. Zusätzlich wurden Zufriedenheit und selbst geschätzte Nutzungshäufigkeit der Lernanwendungen abhängig vom verwendeten mobilen Endgerät der Nutzenden betrachtet. Die Ergebnisse zeigen signifikante Unterschiede zwischen den Einflussfaktoren auf präsenzgebundenes und selbstgesteuertes Mobile Learning auf. Während der empfundene Mehrwert den wichtigsten Einflussfaktor auf die Nutzungsintention beim selbstgesteuerten Mobile Learning darstellt, sind dies im Vorlesungsraum soziale Einflüsse. Die Identifikation dieser grundlegenden Unterschiede stellt den wesentlichen theoretischen Beitrag der Arbeit dar. Zudem deuten die Ergebnisse in Bezug auf die Endgeräte darauf hin, dass innerhalb des Vorlesungssaales Tablet PCs besser für Mobile Learning geeignet sind und außerhalb gegenwärtig noch Laptops. Diese Erkenntnisse bieten Lehrenden Anhaltspunkte über wichtige zu berücksichtigende Einflussfaktoren im Mobile Learning.

Schlüsselbegriffe: Blended Learning, Mobile Learning, Tablet PCs, Nutzerakzeptanz, UTAUT 


\subsection{Motivation und Zielsetzung der Studie}

Mobile Learning verbindet E-Learning mit Mobile Computing durch den Einsatz von Internet, Drahtlosnetzwerken und mobilen Endgeräten (Motiwalla 2007). Mobile Learning ermöglicht damit, Inhalte und Dienste orts- und zeitunabhängig abzurufen und so Pausen oder Leerlaufzeiten effektiver zu nutzen. Zugleich erlaubt es aber genauso, E-Learning-Anwendungen innerhalb von Institutionen wie Unternehmen, Schulen und Universitäten einzusetzen (Sharples et al. 2005). Mobilität entsteht in diesem Falle dadurch, dass sich Lernende innerhalb einer Institution beliebig zwischen unterschiedlichen Räumen und Arbeitsplätzen bewegen können und dabei stets Zugriff auf dieselben Informationen und Anwendungen haben. Dadurch lassen sich Präsenzveranstaltungen um E-Learning-Anwendungen anreichern, was didaktisch äußerst vielversprechend scheint (Duncan-Howell und Lee 2007). Insbesondere in Massenveranstaltungen verbindet sich mit dem Einsatz mobiler Endgeräte die Hoffnung, dem verbreiteten Problem mangelnder Interaktivität zu begegnen, da Übungen auf den Geräten von allen Beteiligten durchgeführt und die Ergebnisse in Echtzeit ausgewertet werden können. In diesem Beitrag werden daher nachfolgend zwei unterschiedliche Formen von Mobile Learning unterschieden: selbstgesteuertes Mobile Learning, das jederzeit und überall ohne eine direkte Kontrolle durch Lehrende stattfindet, sowie präsenzgebundenes Mobile Learning, bei dem mobile Endgeräte und Drahtlosnetzwerke dazu genutzt werden, traditionelle Lernszenarien im Vorlesungssaal zu bereichern.

Während E-Learning an deutschen Universitäten mittlerweile weit verbreitet ist, stellt das Mobile Learning in diesem Kontext jedoch noch einen vergleichsweise neuen Trend dar. Entsprechend ist es nötig, diejenigen Faktoren zu erforschen, welche den didaktisch effektiven Einsatz von Mobile Learning unterstützen, um Schlussfolgerungen für die Konzeption geeigneter Lernszenarien zu ziehen. Denn ein Mehrwert für die Lernenden entsteht nur im Falle einer aktiven Nutzung der Lernanwendungen, da Interaktion eine Basis für Lernerfolg darstellt (Thurmond und Wambach 2004).

Eines der bekanntesten Modelle zur Erklärung der Nutzungsabsicht basiert auf der Unified Theory of Acceptance and Use of Technology (UTAUT) von Venkatesh, Morris, Davis und Davis (2003). Bisherige Studien auf Basis des UTAUTModells im Bereich Mobile Learning adressieren zwar das vollständig selbstgesteuerte Lernen, nicht aber den Einsatz von Mobile Learning innerhalb von Präsenzszenarien. Dies erscheint jedoch zu kurz gegriffen, denn während der Einsatz von Mobile Learning in rein selbstgesteuerten Lernszenarien, zum Beispiel durch Podcasts, vermutlich sehr ähnlich zum klassischen E-Learning verläuft, bietet das Mobile Learning im Vorlesungssaal eine komplett andere Lernumge- 
bung. In diesem Falle haben Lernende unmittelbaren Kontakt zueinander und zum Dozierenden. Sie können Fragen stellen und sehen, dass ihre Kommilitonen/innen dieselben Lernanwendungen wie sie selbst benutzen. Eine explizite Unterscheidung beider Szenarien erscheint damit notwendig, da Aspekte wie Lernumgebung oder zwischenmenschliche Unterstützung wesentlichen Einfluss auf die Wahrnehmung durch die Lernenden ausüben (Benson Soong et al. 2001; Sun et al. 2008). Entsprechend ist davon auszugehen, dass sich auch die Einflussgrößen auf die Nutzungsabsicht deutlich unterscheiden. Die Möglichkeit, im Falle eines Problems schnelle Hilfe durch eine/n Kommilitonen/in oder den Betreuenden einzuholen, ist beispielsweise ausschließlich während der Präsenzveranstaltung gegeben. Unterscheiden sich die Einflussfaktoren tatsächlich deutlich voneinander, legt dies jedoch nahe, dass bisherige Untersuchungsergebnisse zur Nutzungsintention im Mobile Learning nur mit Abstrichen auf den Einsatz innerhalb des Vorlesungssaales übertragen werden können. Eine fehlende Unterscheidung zwischen diesen beiden Szenarien kann demnach zu falschen Schlussfolgerungen führen. Um diese Forschungslücke zu schließen, wurde im Rahmen eines Pilotprojekts die Nutzungsabsicht von Mobile Learning-Anwendungen als reine Selbstlernmaterialien sowie als Aktivierungsübungen im Vorlesungssaal auf Basis des UTAUTModells untersucht.

Das UTAUT-Modell ist gerade im Hinblick auf Aspekte von Technik und Gebrauchstauglichkeit äußerst generisch gehalten. Vor dem Hintergrund der aktuellen Entwicklungen am Hardwaremarkt mit der zunehmenden Verbreitung von Tablet PCs und Smartphones stellt sich jedoch die praktisch relevante Frage, welche Vor- oder Nachteile mit den unterschiedlichen Klassen an Endgeräten verbunden sind. Dies ist gerade wieder vor dem Hintergrund der Unterscheidung zwischen selbstgesteuertem und präsenzgebundenem Mobile Learning von Interesse, da sich mit der neueren Geräteklasse der Tablet PCs die Hoffnung verbindet, dass diese sich deutlich natürlicher in den Vorlesungsraum einfügen (Wegener et al. 2011). Um diese Vermutung zu untersuchen, wird ergänzend zu den Ergebnissen des UTAUT betrachtet, ob sich die wahrgenommene Zufriedenheit und Nutzung der Lernanwendungen zwischen einer Gruppe von Tablet PC-Benutzern/innen und Laptop-Benutzern/innen unterscheiden. 


\subsection{Theoretische Grundlagen zur Nutzungsintention im Mobile Learning}

\subsubsection{TAM und UTAUT als verbreitete Technologieakzeptanzmodelle}

Technologieakzeptanzmodelle befassen sich mit der Frage, welche Faktoren einen besonderen Einfluss darauf haben, ob ein Nutzer ein Informationssystem einsetzen möchte und letztlich auch tatsächlich einsetzt. Ein bekanntes Modell dieser Art stellt das Technology Acceptance Model (TAM) dar (Davis 1989). Das Modell geht davon aus, dass die Nutzungsabsicht gegenüber einem System weitestgehend von zwei Faktoren bestimmt wird, nämlich der wahrgenommenen Nützlichkeit und der Nutzerfreundlichkeit. Obwohl das TAM-Modell immensen Einfluss auf die Forschung hatte, wurde es aufgrund seiner Einfachheit kritisiert. Insbesondere wurde angeführt, dass die durch TAM gemessene Nutzungsintention die letztliche Nutzung nur eingeschränkt erklären kann (Straub und Burton-Jones 2007; Straub et al. 1995). Das TAM-Modell wurde daher mehrmals weiterentwickelt und angepasst. Eine viel beachtete und benutzte Weiterentwicklung stellt UTAUT (Unified Theory of Acceptance and Use of Technology) nach Venkatesh et al. (2003) dar. In diesem Modell werden als wesentliche Einflussgrößen auf die Nutzungsintention (Behavioral Intention) die Performance Expectancy (erwarteter Nutzen des Systems), Effort Expectancy (erwarteter Aufwand zur Nutzung) und Social Influence (soziale Einflüsse, zum Beispiel durch Vorgesetzte oder Kollegen/innen) identifiziert. Als Facilitating Conditions werden schließlich weitere unterstützende oder hinderliche Faktoren wie die zur Einarbeitung zur Verfügung stehende Zeit oder der Support erfasst. Die ersten drei Variablen beeinflussen dabei die Intention zur Nutzung des Systems, letztere die tatsächliche Nutzung. Zudem existieren die moderierenden Variablen Geschlecht, Alter, Erfahrung und Freiwilligkeit der Nutzung. Eine vereinfachte Darstellung ohne die moderierenden Variablen zeigt Abb. 6.1. Da das UTAUT-Modell in unterschiedlichen Kontexten eine hohe Erklärungskraft besitzt, wird es auch im Rahmen dieser Studie als Grundlage herangezogen. 
Abb. 6.1 Vereinfachtes UTAUT-Modell in Anlehnung an Venkatesh u.a. (2003)

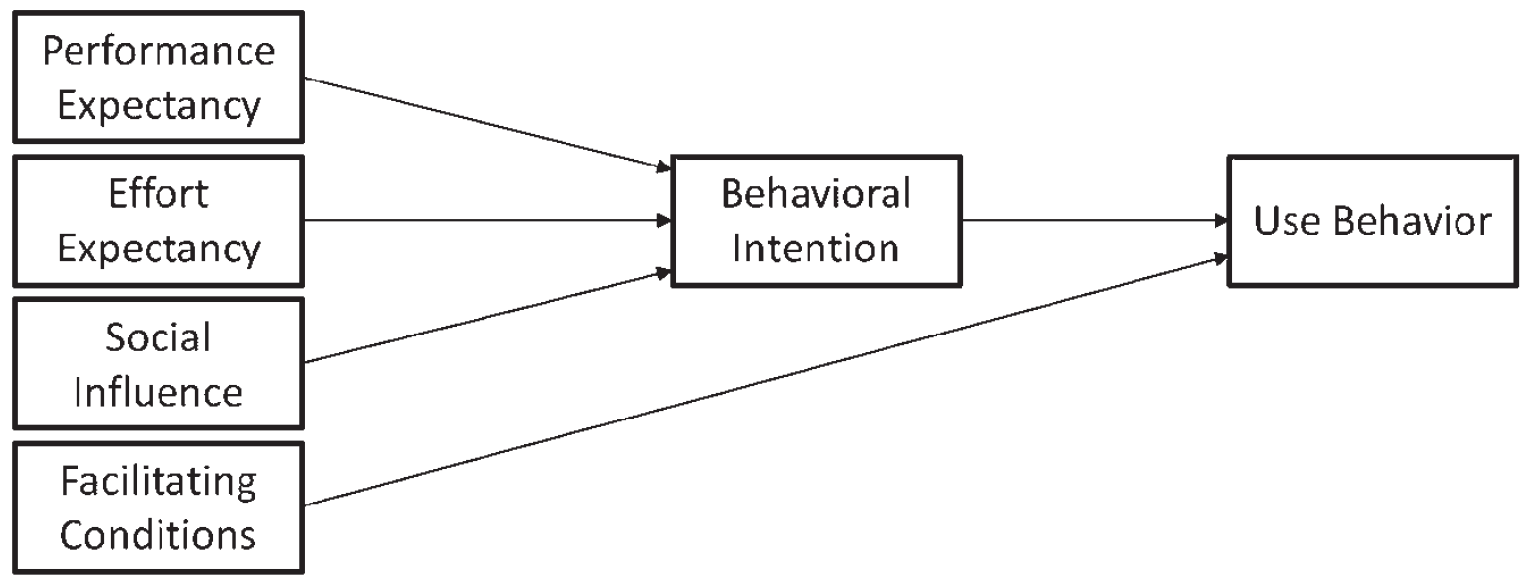

\subsubsection{Anwendung des UTAUT-Modells im Mobile Learning}

Das UTAUT-Modell ist zunächst unabhängig von einer konkreten Problemdomäne und lässt sich auf verschiedene Bereiche wie eben auch das E-Learning oder Mobile Learning übertragen. In letzterem Bereich konnten über eine Recherche in den Datenbanken IEEE XPlore, ScienceDirect, AIS.Net, Ebsco Host und ERIC (Educational Resources Information Center) mittels des Suchstring „UTAUT Mobile Learning“ insgesamt drei empirische Arbeiten identifiziert werden. Diese beschäftigen sich mit dem allgemeinen Einsatz von Tablet PCs (El-Gayar und Moran 2006), Audio-Podcasts (Ho und Chou 2009) sowie Mobile Learning im Allgemeinen (Wang et al. 2009). In allen Studien kommen modifizierte Fassungen des UTAUT-Modells zum Einsatz, es wird jedoch niemals präsenzgebundenes Mobile Learning adressiert sondern stets das selbstgesteuerte. Die Ergebnisse der genannten Studien zeigen dabei einheitlich, dass der erwartete Mehrwert (Performance Expectancy) den wichtigsten Faktor für die Lernenden darstellt (Abb. 6.2), während soziale Einflüsse lediglich eine eher untergeordnete Rolle spielen. 
Abb. 6.2 Zusammenfassung bisheriger Studien zur Nutzungsintention im Mobile Learning

\begin{tabular}{|l|l|l|l|}
\hline Autor & $\begin{array}{l}\text { Erwarteter } \\
\text { Mehrwert }\end{array}$ & Aufwand & Soziale Einflüsse \\
\hline $\begin{array}{l}\text { E-Gayar \& Mora 2006: Einsatz von Tablet } \\
\text { PCs allgemein }\end{array}$ & 0,19 & 0,12 & 0,06 \\
\hline Ho \& Chou 2009: Audio-Podcasts & Most Important* & & $\begin{array}{l}\text { Second } \\
\text { Important* }\end{array}$ \\
\hline $\begin{array}{l}\text { Wang et al. 2009: Mobile Learning allge- } \\
\text { mein }\end{array}$ & 0,26 & 0,21 & 0,12 \\
\hline
\end{tabular}

*konkrete Zahlen liegen nicht vor

Die betrachteten Studien haben jedoch den Nachteil, dass sie vergleichsweise allgemeine Einsatzszenarien beschreiben und nicht eine konkrete Lernanwendung innerhalb eines Seminars. Eine Unterscheidung zwischen selbstgesteuertem und präsenzgebundenem Einsatz findet ebenso wenig statt. Damit stellt sich die Frage, ob die Ergebnisse tatsächlich in die universitäre Praxis übertragen werden können.

\subsubsection{Forschungsfragen des Beitrags}

Das primäre Ziel dieses Beitrags besteht folglich in einer differenzierten Betrachtung der Einflussfaktoren auf die Nutzungsintention innerhalb eines konkreten Einsatzszenarios. Dabei wird zusätzlich zwischen selbstgesteuertem und präsenzgebundenem Mobile Learning im Vorlesungssaal unterschieden. Als sekundäres Ziel wird zudem untersucht, ob sich die Wahrnehmung der beiden Anwendungskontexte aufgrund der eingesetzten Endgeräteklasse (Laptop beziehungsweise Tablet PC) unterscheidet.

Damit werden die drei nachfolgend vorgestellten Forschungsfragen beantwortet.

1. Was sind Einflussgrößen auf die Nutzungsabsicht von Mobile Learning und inwieweit stimmen diese mit bisherigen Studien überein?

2. Welche Unterschiede bezüglich der Einflussgrößen auf die Nutzungsabsicht können zwischen selbstgesteuertem und präsenzgebundenem Mobile Learning beobachtet werden?

3. Welcher Zusammenhang besteht zwischen der Klasse des Endgerätes und der Nutzung von Zufriedenheit mit Mobile Learning-Anwendungen in beiden Anwendungskontexten? 


\subsection{Fallstudie und Datenerhebung}

\subsubsection{Einsatzszenario}

Im vorliegenden Fall wurden im Rahmen eines Pilotprojekts im Wintersemester 2010/2011 rund 150 Tablet PCs in Form von Apple iPads für ein Semester an Bachelorstudierende der Wirtschaftswissenschaften verliehen, die eine Lehrveranstaltung zur Einführung in die Wirtschaftsinformatik besuchten. Insgesamt nahmen ca. 250 Studierende an der Pflichtlehrveranstaltung teil. Jeder von ihnen konnte sich um die Ausleihe eines solchen Tablet PCs bewerben. Da zudem ein Großteil der Studierenden bereits über andere mobile Endgeräte, vorwiegend Laptops, verfügte, und weitere 50 Netbooks zur Ausleihe zur Verfügung standen, konnte erstmalig eine komplette Abdeckung aller Studierenden dieser Lehrveranstaltung mit mobilen Endgeräten erreicht werden. Insgesamt bewarben sich knapp 150 Teilnehmende für die Ausleihe eines Tablet PCs und einige wenige für die Ausleihe eines Netbooks. Alle Teilnehmenden konnten mit den von ihnen gewünschten Endgeräten ausgestattet werden. Damit war sichergestellt, dass alle Teilnehmenden die zur Verfügung gestellten Mobile Learning-Anwendungen nutzen konnten.

In der Veranstaltung wurde Mobile Learning im Rahmen zweier Anwendungskontexte erprobt. Zum einen wurden Selbstlernmaterialien in Form von Videoaufzeichnungen und Web Based Trainings zur Verfügung gestellt. Die Videoaufzeichnungen stellen Streams der Vorlesung dar, welche keine zusätzlichen Funktionen aufweisen. Sie dienen in erster Linie dazu, Vorlesungen nachzubereiten oder verpasste Vorlesungen aufzuarbeiten. Die Web Based Trainings stellen speziell auf die Tablet PCs angepasste, in sich geschlossene E-Learning-Einheiten dar (s. Abb. 6.3). Diese behandeln einzelne Themen der Vorlesung und enthalten im Gegensatz zu einem traditionellen Skript Animationen und interaktive Übungen.

Daneben wurden während der Präsenztermine Anwendungen zur Aktivierung der Teilnehmenden eingesetzt. Hierbei handelte es sich zum einen um eine Abstimmungssoftware namens „Peer Discussion“, die auf dem Konzept der Peer Instruction basiert (Crouch und Mazur 2001). Dabei wird den Studierenden vom Dozierenden eine Frage mit mehreren möglichen Antworten nach dem MultipleChoice-Prinzip vorgegeben. Diese erhalten nun kurze Bedenkzeit und geben danach ihre Antwort über die Endgeräte ab. Anschließend erhalten sie einige Minuten Zeit, um sich mit ihren Nachbarn über ihre Antwort auszutauschen und diese zu begründen. Dann dürfen sie ihre Meinung bei Bedarf revidieren und erneut 
abstimmen. Auf diesem Wege wird der Austausch der Studierenden untereinander gefördert. Die Ergebnisse werden zugleich vom Dozierenden aufgegriffen, um mögliche Verständnisschwierigkeiten seitens der Studierenden zu identifizieren.

\section{Abb. 6.3 Beispiele für Web Based Trainings}
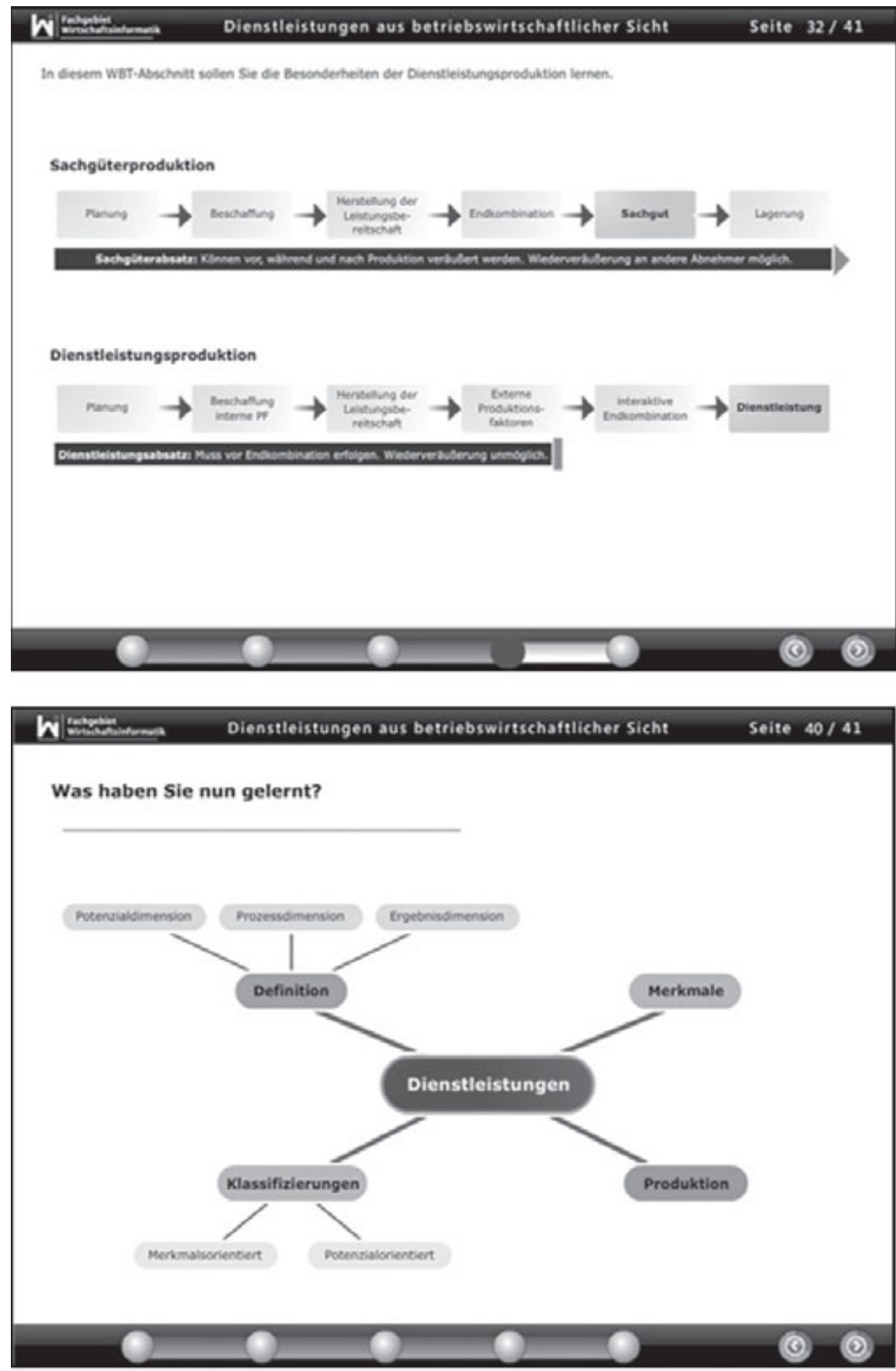
Als zweite Form der Aktivierung wurden die Studierenden während der Vorlesung aufgefordert, Wahr-Falsch-Aussagen zu den Inhalten der Veranstaltung zu generieren, welche sie elektronisch abgeben konnten. Das Erstellen dieser Aussagen dient zunächst der Reflektion der vorangegangenen Inhalte und ist zugleich als kurze Erholungsphase gedacht (Olmsted III 1999). Die eingegebenen Aussagen werden anschließend an eine Datenbank übertragen und wieder unter den Studierenden verteilt, sodass diese jeweils drei Aussagen ihrer Kommilitonen lösen und bewerten können. Von den am besten bewerteten Aussagen werden einige vom Dozierenden ausgewählt, die später in der Klausur eingesetzt werden, weshalb die Übung „Co-Create Your Exam“ genannt wird. Zudem werden fünf Aussagen exemplarisch vom Dozierenden innerhalb der Vorlesung gelöst (s. Abb. 6.4).

\section{Abb. 6.4 Übung „Co-Create Your Exam“}

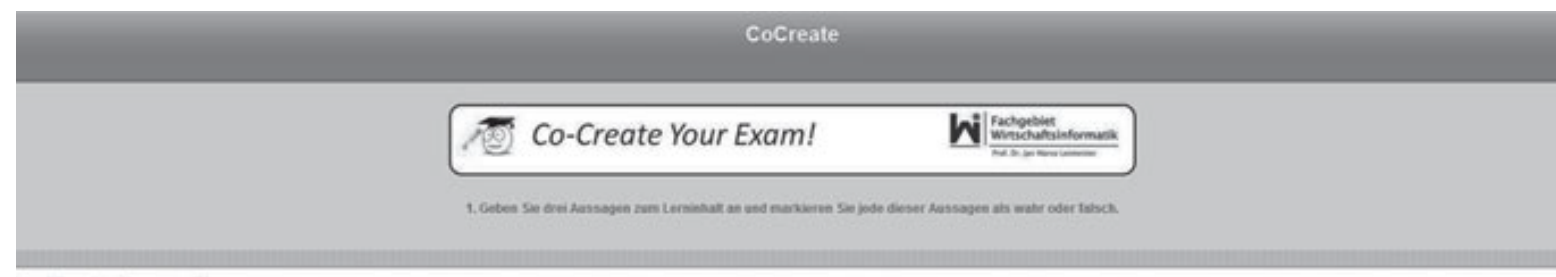

jer dio ersto Aussage ein

Beide Aktivierungsübungen kamen jeweils einmal innerhalb von zehn Präsenzveranstaltungen zum Einsatz. Einen Überblick über alle eingesetzten Mobile Learning-Anwendungen liefert die Abb. 6.5.

\subsubsection{Datenerhebung}

Am Ende des Semesters, jedoch vor der Klausur, wurden zwei Online-Fragebögen zur Evaluation eingesetzt. In der ersten Befragung lag der Fokus auf der Nutzungsabsicht, wohingegen die zweite Befragung die persönlich wahrgenommene Lernendenzufriedenheit stärker thematisiert. Beide Befragungen standen für einen Zeitraum von mehreren Tagen online zur Verfügung. Die Studierenden wurden per Mail und in der Vorlesung darüber informiert. Die Teilnahme erfolgte freiwillig und anonym. 


\section{Abb. 6.5 Übersicht über die eingesetzten Mobile Learning-Anwendun-} gen

\begin{tabular}{|c|c|c|}
\hline Einsatz & Anwendung & Beschreibung \\
\hline & Co-Create Your Exam & $\begin{array}{l}\text { Studierende erstellen in der Vorlesung innerhalb weniger Minuten } \\
\text { drei Wahr-Falsch-Aussagen zu den vorangegangenen Inhalten. } \\
\text { Anschließend lösen sie die Aufgaben ihres Nachbarn. Fünf zufäl- } \\
\text { lig gewählte Aussagen werden vom Dozierenden vorgeführt und } \\
\text { beispielhaft gelöst. }\end{array}$ \\
\hline 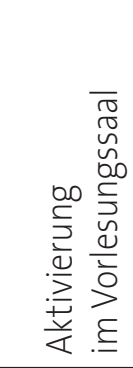 & Peer Discussion & $\begin{array}{l}\text { Studierende entscheiden sich, welche von fünf gegebenen } \\
\text { Aussagen die richtige ist und stimmen ab. Anschließend disku- } \\
\text { tieren Sie ihre Lösung mit dem/der Nachbarn/in und dürfen ihre } \\
\text { Meinung ändern. Die Ergebnisse der Abstimmung werden mit- } \\
\text { tels Beamer für alle sichtbar dargestellt und vom Dozierenden } \\
\text { aufgegriffen. }\end{array}$ \\
\hline \multirow{2}{*}{ 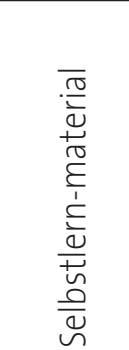 } & Web Based Trainings & $\begin{array}{l}\text { Einzelne Lerneinheiten von ca. } 30 \text { Minuten Länge, die einzelne } \\
\text { Themen der Vorlesung aufgreifen. Die Trainings verwenden } \\
\text { Animationen und interaktive Übungen zur besseren Vermittlung } \\
\text { von Inhalten. }\end{array}$ \\
\hline & Videostream & $\begin{array}{l}\text { Die Vorlesung wird aufgezeichnet und als Videostream sowohl live } \\
\text { als auch dauerhaft im Internet zur Verfügung gestellt. }\end{array}$ \\
\hline
\end{tabular}

Der erste Fragebogen zur Evaluierung der Nutzungsintention basierte auf dem UTAUT-Modell nach Venkatesh u.a. (2003). Es wurden lediglich die einzelnen UTAUT-Items ins Deutsche übersetzt und auf den Lernbereich angepasst. Der Fragebogen enthielt einen Teil zum selbstgesteuerten Einsatz (Videos und Web Based Trainings) sowie einen Teil zur Aktivierung während der Präsenzveranstaltungen (Peer Discussion und Co-Create Your Exam), um beide Anwendungskontexte miteinander vergleichen zu können. Insgesamt füllten 129 Studierende den Fragebogen vollständig aus. Bezogen auf die Anzahl von 239 Klausurteilnehmenden entspricht dies einer Teilnahmequote von ca. 54 \%. Auf Basis der Fragebögen wurden Einflussfaktoren auf die Nutzungsabsicht bestimmt. Aus technischen Gründen konnte jedoch die tatsächliche Nutzung nicht auf aussagekräftige Weise erfasst werden, da es sich beispielsweise bei den Aktivierungen um WebApplikationen handelte, die ohne Login abrufbar waren, um einen reibungslosen Zugriff während der Vorlesung zu ermöglichen. Dies machte eine Zuordnung der 
Aufrufe zu konkreten Studierenden aber unmöglich. Daher wurde im Gegensatz zum ursprünglichen Modell auch der Einfluss der unterstützenden Faktoren auf die Nutzungsabsicht anstelle der tatsächlichen Nutzung bestimmt.

Im Rahmen der zweiten Online-Befragung wurden die Studierenden zu ihrer Einschätzung und Zufriedenheit mit verschiedenen Elementen der Lehrveranstaltung befragt. Die von uns entwickelten Items orientieren sich an wesentlichen Dimensionen der Lernendenzufriedenheit wie Struktur und Schwierigkeitsgrad der Veranstaltung oder den wahrgenommenen Fähigkeiten des Dozierenden (Cohen 1981). Die Items einer Kategorie sind jeweils als Aussagen oder Fragen formuliert, deren Zustimmung auf einer 5er Likert-Skala abgefragt wird, beispielsweise „Die WBTs fördern die Nachbereitung der Inhalte aus der Vorlesung“ oder „Wie zufrieden sind Sie mit der Veranstaltung generell?“. Die Zustimmung wird auf der Likert-Skala von „trifft zu“ bis „trifft nicht zu“ beziehungsweise „sehr zufrieden“ bis ,gar nicht zufrieden“ eingetragen. An dieser freiwilligen Befragung haben 128 Studierende teilgenommen, was erneut in etwa einer Teilnahmequote von $54 \%$ entspricht. In der Grundgesamtheit von $n=128$ enthalten sind 66 Studierende, die einen Tablet PC genutzt haben und 62 Studierende, bei denen dies nicht der Fall war. Die Angaben über den Einsatz eines Tablet PCs oder Laptops basieren auf der Selbstauskunft der Studierenden. Nachfolgend werden die Ergebnisse erläutert, wobei im Rahmen dieser Arbeit der Fokus auf die Dimensionen zur allgemeinen Zufriedenheit mit der Lehrveranstaltung sowie die spezifischen Lernanwendungen gerichtet ist.

\subsection{Ergebnisse und Diskussion}

\subsubsection{Einflussgrößen auf die Nutzungsabsicht von Mobile Learning}

Was sind Einflussgrößen auf die Nutzungsabsicht von Mobile Learning und inwieweit stimmt dies mit bisherigen Studien überein?

Vor der Betrachtung der Einflussfaktoren auf die Nutzungsabsicht ist eine Überprüfung der für die Messmodelle relevanten Qualitätskriterien nach Chin (1998) notwendig. Hierfür wurden die durchschnittlich erfasste Varianz (DEV), die Konstruktreliabilität, die Kreuzladungen der einzelnen Indikatoren und die Diskriminanzvalidität untersucht.

Bei den Selbstlernmaterialien lagen alle DEV-Werte über dem Grenzwert von 0,5. Auch die Konstruktreliabilität lag bei allen Konstrukten deutlich über dem Grenzwert von 0,6. Die Kreuzladungen der einzelnen Indikatoren waren 
stets niedriger als die Ladungen zwischen den Indikatoren und den zugeordneten Konstrukten, ebenso wurde die Diskriminanzvalidität bewiesen, da der jeweilige DEV-Wert durchweg höher war als die Korrelation einer latenten Variable mit den anderen Variablen.

Auch bei den Aktivierungsübungen lagen alle DEV-Werte über dem Grenzwert von 0,5, und auch die Konstuktreliabilität lag in allen Fällen über dem Grenzwert von 0,6. Die Kreuzladungen der Indikatoren waren stets niedriger als die Ladungen zwischen den Indikatoren und den entsprechenden Konstrukten. Da auch der jeweilige DEV-Wert höher war als die Korrelation einer Variablen mit den anderen Variablen, gilt die Diskriminanzvalidität als erwiesen. Somit erfüllen die Messmodelle die Qualitätskriterien, was eine Analyse der strukturellen Zusammenhänge ermöglicht. Bei den Aktivierungsübungen war der T-Wert bei den unterstützenden Bedingungen nicht signifikant, die übrigen T-Werte zeigen hingegen einen hochsignifikanten Einfluss der Konstrukte an $(p<0,01)$. Damit kann eine Varianz von $52 \%$ der Nutzungsabsicht erklärt werden, was nach Chin (1998) zwischen dem moderaten und substanziellen Niveau liegt. Beim Selbstlernmaterial sind die T-Werte für alle Konstrukte mindestens signifikant ( $\mathrm{p}<0,05)$, womit sich $47 \%$ der Varianz der Nutzungsabsicht erklären lassen und so wiederum ein moderates bis substanzielles Niveau erreicht wird. Abb. 6.6 veranschaulicht die zuvor diskutierten Zusammenhänge nochmals zusammengefasst. Das UTAUT-Modell ist somit im vorliegenden Fall geeignet, Einflussfaktoren auf die Nutzungsabsicht zu erklären und zur Analyse der Forschungsfragen beizutragen (s. Abb. 6.6).

Bezüglich des Einflusses der einzelnen Faktoren zeigen sich deutliche Unterschiede zwischen beiden Anwendungskontexten. Wie bereits in Abschnitt 2 dargelegt, deuten bisherige Ergebnisse einheitlich darauf hin, dass der erwartete Mehrwert eine zentrale Determinante für die Nutzungsabsicht von Mobile Learning ist, was die Ergebnisse der vorliegenden Untersuchung für das selbstgesteuerte Lernen bestätigen. Auch kann analog zu El-Gayar und Moran (2006) und Wang et al. (2009) bestätigt werden, dass der erwartete Aufwand den zweitwichtigsten Faktor darstellt. Auffällig ist im vorliegenden Fall der besonders hohe Einfluss des erwarteten Mehrwerts, sowohl im Rahmen dieser Studie als auch im Vergleich mit den anderen.

Dies kann möglicherweise damit begründet werden, dass es sich hier um einen konkreten Anwendungsfall handelt, in dem die Lernmaterialien im Unterschied zu den anderen Studien explizit als Zusatzmaterialien deklariert sind. Für deren freiwillige Bearbeitung erwarten die Studierenden im Gegenzug in besonderem Maße einen hohen Nutzen. Der erwartete Mehrwert scheint insgesamt über viele selbstgesteuerte Anwendungskontexte hinweg das zentrale Argument für die Nutzung von selbstgesteuertem Mobile Learning darzustellen. 
Abb. 6.6 PLS-Modell zu Erklärung der Nutzungsabsicht

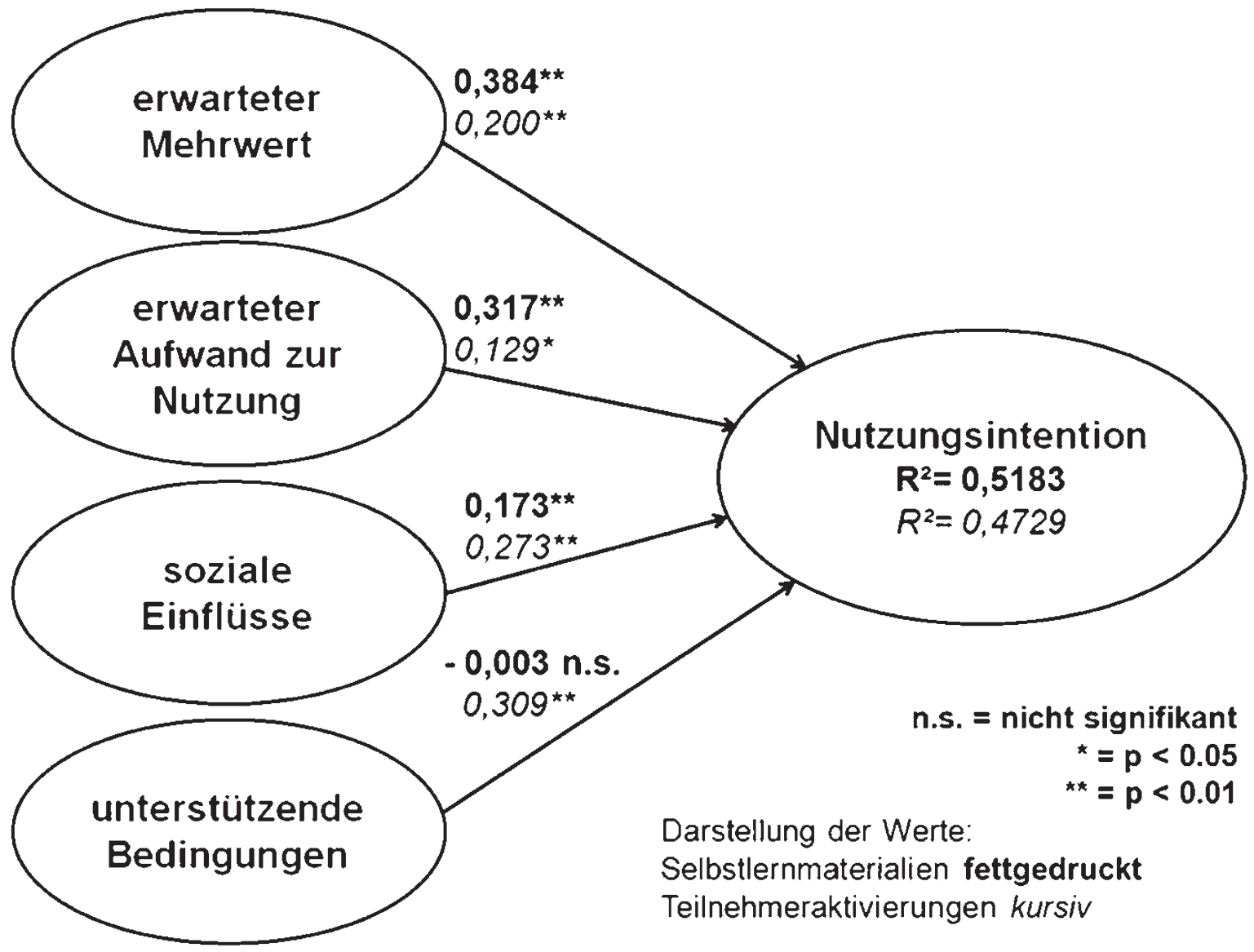

Diese Erkenntnis gilt jedoch nicht für die Anwendung innerhalb des Vorlesungssaales, wo die Einflüsse der Faktoren deutlich anders gewichtet sind.

\subsubsection{Vergleich der unterschiedlichen Anwendungskontexte}

Welche Unterschiede bezüglich der Einflussgrößen auf die Nutzungsabsicht können zwischen selbstgesteuertem und präsenzgebundenem Mobile Learning beobachtet werden?

Die Ergebnisse zeigen, dass sich im vorliegenden Fall die Determinanten der Nutzungsabsicht des in Präsenzveranstaltungen eingebetteten Mobile Learnings deutlich von denen des selbstgesteuerten unterscheiden. Zwar stellt der erwartete Mehrwert einen wichtigen Faktor dar, zentraler sind jedoch soziale Einflüsse, die in einem durch Dozierende geprägten Lernszenario in den Mittelpunkt rücken. Die Lernanwendungen werden folglich dann genutzt, wenn die oder der Dozierende 
explizite Beteiligung erbittet und durch Hilfestellungen erleichtert. Von zentraler Bedeutung sind zudem die unterstützenden Faktoren wie ausreichend Zeit für die Nutzung der Anwendungen und umfassende Erläuterungen.

Die Ergebnisse sind ein Hinweis darauf, dass für Lernanwendungen, die in Präsenzphasen zum Einsatz kommen, technische und konkrete inhaltliche Aspekte weniger wichtig sind, als man dies annehmen könnte. Vielmehr steht stattdessen die Frage nach der sinnvollen Einbettung in die Veranstaltung im Vordergrund. Klare Anweisungen und Erwartungen, ausreichend Zeit und wenn nötig Hilfestellungen scheinen die Nutzungsintention stärker zu beeinflussen.

\subsubsection{Einfluss der Art des Endgerätes auf wahrgenommene Zufriedenheit und Nutzung der Anwendungen}

Inwiefern besteht ein Zusammenhang zwischen der Art des Endgerätes und der Nutzung von/Zufriedenheit mit Mobile Learning-Anwendungen?

Die zweite Online-Befragung fokussierte auf die Nutzung und Zufriedenheit mit den angebotenen Mobile Learning-Anwendungen. Die nachfolgenden Ergebnisse basieren auf diesen zugrunde liegenden Daten. Dabei wurden die Mittelwerte in beiden Vergleichsgruppen (Tablet PC und Laptop) analysiert. Bei der Datenauswertung wurde zunächst geprüft, ob die Mittelwerte normal verteilt sind. In diesem Kontext zeigte der Kolmogorov-Smirnov-Test, dass die Daten nicht normal verteilt sind. Folglich ist zur Untersuchung der Signifikanzen ein nichtparametrischer Test nötig. Im Zuge der weiteren Analyse kam daher der Wilcoxon-Mann-Whitney-U-Test zur Anwendung. Nachfolgend sind die Testvariablen des Fragebogens in verkürzter Form dargestellt. Die Variablen sind dabei kategorisiert in die Bereiche Zufriedenheit mit der Struktur der Lehrveranstaltung, wahrgenommene Interaktivität, Zufriedenheit mit dem Selbstlernmaterial (unterteilt in Web Based Trainings und Videos) sowie Zufriedenheit mit den Aktivierungsübungen während der Vorlesung (unterteilt in Co-Create Your Exam und Peer Discussion). Neben den jeweiligen Mittelwerten der Gruppen zeigt die Tabelle auch die Standardabweichung sowie die Signifikanz der Unterschiede der Mittelwerte. Signifikante Werte sind mit einem Stern markiert. Neben den direkt abgefragten Items wird zudem zur besseren Übersichtlichkeit ein Mittelwert der einzelnen Kategorien angegeben (Abb. 6.7). 
Abb. 6.7 Zufriedenheit der Studierenden, unterschieden nach genutztem Endgerät

\begin{tabular}{|c|c|c|c|c|c|c|c|}
\hline \multirow{2}{*}{\multicolumn{2}{|c|}{ 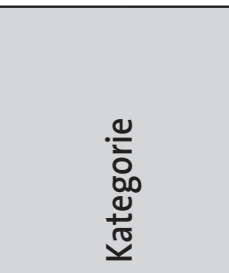 }} & \multirow[t]{2}{*}{ Item } & \multicolumn{2}{|c|}{ Tablet PC $(n=66)$} & \multicolumn{2}{|c|}{ Laptop $(n=62)$} & \multirow[b]{2}{*}{ 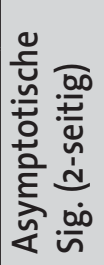 } \\
\hline & & & 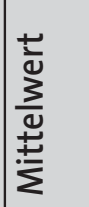 & 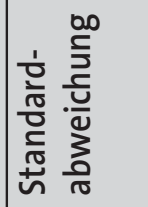 & 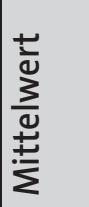 & 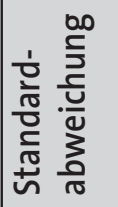 & \\
\hline \multirow{6}{*}{\multicolumn{2}{|c|}{ 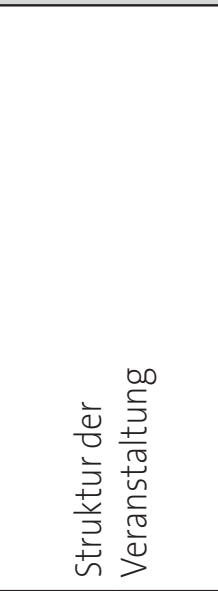 }} & Thematische & 2.74 & 0.87 & 2.65 & 1.12 & 0.510 \\
\hline & & $\begin{array}{l}\text { Struktur und Roter } \\
\text { Faden }\end{array}$ & 2.05 & 0.89 & 1.90 & 0.76 & 0.429 \\
\hline & & $\begin{array}{l}\text { Verhältnis Inhalt/ } \\
\text { Aufwand }\end{array}$ & 3.23 & 1.02 & 3.34 & 1.10 & 0.569 \\
\hline & & Schwierigkeitsgrad & 3.30 & 1.07 & 3.15 & 0.96 & 0.307 \\
\hline & & $\begin{array}{l}\text { Verhältnis Inhalt } \\
\text { und Zeit }\end{array}$ & 3.36 & 1.02 & 3.52 & 1.05 & 0.373 \\
\hline & & $\begin{array}{l}\text { Transparenz und } \\
\text { Leistung } \\
\text { Mittelwert }\end{array}$ & $\begin{array}{l}2.41 \\
2.85\end{array}$ & 1.05 & $\begin{array}{l}2.45 \\
2.84\end{array}$ & 0.97 & 0.604 \\
\hline \multirow{2}{*}{\multicolumn{2}{|c|}{ 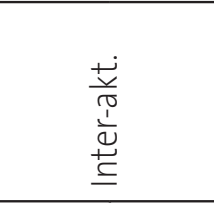 }} & Interaktivität & 2.00 & 0.93 & 2.27 & 0.94 & 0.069 \\
\hline & & $\begin{array}{l}\text { Studentische } \\
\text { Beteiligung } \\
\text { Mittelwert }\end{array}$ & $\begin{array}{l}2.48 \\
2.24\end{array}$ & 1.09 & $\begin{array}{l}2.82 \\
2.55\end{array}$ & 1.26 & 0.130 \\
\hline \multirow{14}{*}{ 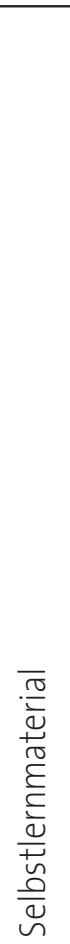 } & \multirow{8}{*}{ 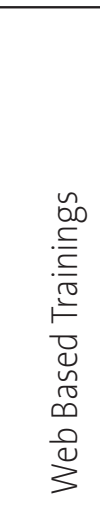 } & Aufbereitung & 2.11 & 0.93 & 2.11 & 0.96 & 0.950 \\
\hline & & Interaktivität & 2.12 & 0.87 & 2.06 & 0.90 & 0.666 \\
\hline & & $\begin{array}{l}\text { Hilfe zur Nach- } \\
\text { bereitung }\end{array}$ & 2.05 & 0.81 & 1.98 & 0.97 & 0.517 \\
\hline & & $\begin{array}{l}\text { Individuelles } \\
\text { Lerntempo }\end{array}$ & 2.23 & 0.84 & 2.16 & 0.94 & 0.677 \\
\hline & & Individueller Lernstil & 2.35 & 0.85 & 2.45 & & 0.640 \\
\hline & & Sinnvolle Ergänzung & 2.02 & 0.87 & 2.06 & & 0.874 \\
\hline & & Aktive Nutzung & 2.61 & 0.93 & 2.63 & & 0.848 \\
\hline & & Mittelwert & 2.21 & & 2.21 & & \\
\hline & \multirow{6}{*}{$\begin{array}{l}\tilde{0} \\
\frac{d}{0} \\
\frac{0}{>}\end{array}$} & $\begin{array}{l}\text { Aufbereitung/ } \\
\text { Qualität }\end{array}$ & 2.39 & 1.14 & 2.29 & & 0.664 \\
\hline & & $\begin{array}{l}\text { Individuelles } \\
\text { Lerntempo }\end{array}$ & 2.32 & 1.14 & 1.95 & 1.05 & 0.053 \\
\hline & & Individueller Lernstil & 2.21 & 1.14 & 1.98 & 1.22 & 0.136 \\
\hline & & $\begin{array}{l}\text { Hilfe zur } \\
\text { Nachbereitung* }\end{array}$ & 2.00 & 0.99 & 1.68 & 0.94 & 0.041 \\
\hline & & Aktive Nutzung* & 2.61 & 1.33 & 1.81 & 1.16 & 0.000 \\
\hline & & Mittelwert & 2.31 & & 1.94 & & \\
\hline
\end{tabular}




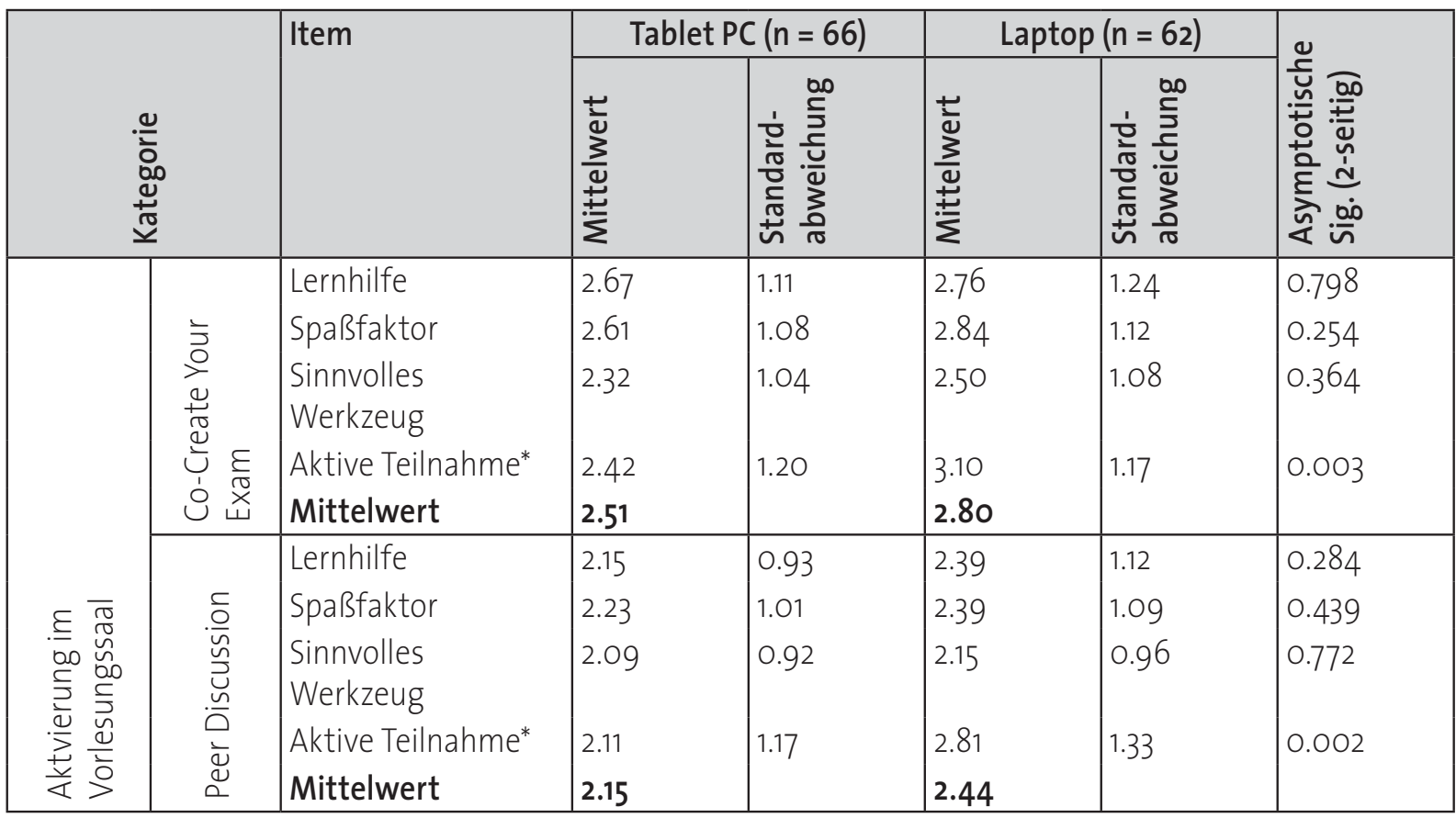

Unter die Kategorie Selbstlernmaterial fallen die eingesetzten Web Based Trainings sowie die Videomitschnitte. Die Web Based Trainings weisen dabei im Gesamtmittelwert keinen Unterschied zwischen den Vergleichsgruppen auf. Auch die einzelnen Items zeigen keine signifikanten Unterschiede. Mit einem Mittelwert von 2,2 ist festzuhalten, dass die Web Based Trainings insgesamt eher positiv wahrgenommen wurden. Ein anderes Bild zeigen die Videos. Diese werden im Schnitt von der Vergleichsgruppe der Laptop-Nutzenden positiver wahrgenommen. Dies zeigt sich bei allen entsprechenden Items, bei den Items „Aktive Nutzung der Videos“ und „Videos sind hilfreich bei der Nachbereitung der Vorlesung“" sogar auf signifikantem Niveau. Dieses Ergebnis erscheint zunächst überraschend, zumal Tablet PCs eigentlich sehr gut dafür geeignet sind, komfortabel Videos abzurufen und zu betrachten. Die wahrgenommene Qualität der Videos unterscheidet sich zwischen den beiden Gruppen zudem kaum. Das deutet darauf hin, dass die Gründe für die unterschiedliche Wahrnehmung weniger im Video an sich liegen könnten, sondern eher in der Möglichkeit, dieses sinnvoll in den eigenen Lernprozess zu integrieren. So bieten die eingesetzten Tablet PCs keine Möglichkeit zu echtem Multi-Tasking, ein Video kann folglich nur für sich alleine betrachtet werden, ohne dass beispielsweise parallel Folien annotiert oder andere Anwendungen genutzt werden. Dies könnte einen wichtigen Nachteil darstellen, zumal ein 90-minütiges Veranstaltungsvideo gegebenenfalls erst in Kombination mit anderen Materialien sinnvoll genutzt werden kann. Im Falle der Web Based Trainings ist dies nicht der Fall, da diese für sich ein hohes Maß an Interaktivität bieten und in sich geschlossen sind, hier ist also die Verwendung zusätzli- 
cher Materialien vermutlich weniger hilfreich. Der genannte Nachteil von Videos könnte jedoch mit wachsenden Fähigkeiten von Tablet PCs schnell wieder ausgeglichen werden. Zudem ist zu berücksichtigen, dass die Web Based Trainings auf den Tablet PCs installiert werden konnten, wohingegen die Videos lediglich als Stream abrufbar waren. Damit sind die Web Based Trainings für das Mobile Lernen auch ohne Internetzugang geeignet, was einen weiteren möglichen Grund für deren verstärkte Nutzung auf Tablet PCs im Vergleich zum Video darstellen könnte.

Bereits die Ergebnisse der Kategorie Interaktivität deuten an, dass Tablet PCs gut zur Förderung derselben eingesetzt werden können. Die Ergebnisse zu den spezifischen Aktivierungsübungen unterstützen diese Vermutung. Sämtliche Items dieser Kategorie fallen bei der Vergleichsgruppe mit Tablet PCs positiver aus, größtenteils allerdings nicht auf signifikantem Niveau. Lediglich die Items zur tatsächlichen Nutzung (aktiven Teilnahme) zeigen besonders starke und statistisch signifikante Unterschiede. Dies bekräftigt die Vermutung, dass Tablet PCs ideal für kurzfristige Aktivierungen geeignet sind. Die Vorteile des permanenten Standby, schnellen Zugangs zum WLAN im Hörsaal und generell kurzer Ladezeiten sind in diesem Fall vermutlich förderlich. Da auch Items wie der Spaßfaktor der Anwendungen bei den Nutzenden von Tablet PCs tendenziell positiver ausfallen, könnte man zudem vermuten, dass die hohe Nutzerfreundlichkeit derselben einen zusätzlichen positiven Effekt auf die Wahrnehmung der Lernanwendungen seitens der Studierenden ausübt. Der Vergleich der Ergebnisse zwischen beiden eingesetzten Anwendungen zeigt zudem, dass die Peer Discussion positiver als Co-Create Your Exam bewertet wurde. Ein wesentlicher Grund hierfür könnte in deren besonders einfacher Handhabung liegen, welche den schnellen Einsatz innerhalb der Lehrveranstaltung begünstigt.

Insgesamt deuten die Ergebnisse also zumindest an, dass es einen Zusammenhang zwischen der Wahl des mobilen Endgerätes und Zufriedenheit beziehungsweise Nutzungshäufigkeit mobiler Lernanwendungen gibt. Inwiefern ein Gerät positiven oder negativen Einfluss ausübt, hängt dementsprechend vermutlich von dessen Eignung für die jeweilige Aufgabe ab. Die eingesetzten Tablet PCs scheinen besonders gut für Anwendungen geeignet, die direkt innerhalb der Vorlesung genutzt werden, da hierbei leichte Bedienbarkeit und Schnelligkeit wesentlich für den sinnvollen Einsatz sind. Dadurch, dass die Geräte zudem ohne aufklappbaren Bildschirm auskommen und äußerst leise arbeiten, ermöglichen sie vermutlich ein „,natürlicheres“ Erlebnis im Hörsaal, was die Ergebnisse ebenfalls erklären könnte. Laptops scheinen im vorliegenden Fall bei der Nutzung von Videos als Selbstlernmaterial Vorteile aufzuweisen. Dies liegt möglicherweise daran, dass diese Form von Lernmaterial aufgrund des Umfangs und der nötigen Internetanbindung für 
jederzeitiges Mobile Learning nicht so gut geeignet ist wie die Web Based Trainings. Zudem ist die Videonutzung gegebenenfalls sinnvoller in Kombination mit anderen Lernmaterialien oder Werkzeugen, beispielsweise zur Annotation von Vorlesungsfolien, was aufgrund des mangelnden Multi-Taskings der eingesetzten Tablet PCs für die Lernenden nicht möglich war. Eine mögliche Lösung könnte in diesem Falle jedoch bereits darin bestehen, Vorlesungsmitschnitte in mehrere kleine Videos zu untergliedern und zum Download anzubieten.

\subsection{Fazit, Einschränkungen und Ausblick}

In der vorliegenden Arbeit wurden Einflussfaktoren auf die Nutzungsintention mobiler Lernanwendungen in zwei Anwendungskontexten untersucht. Dabei handelt es sich um das selbstgesteuerte sowie das präsenzgebundene Mobile Learning. Auf Basis des UTAUT-Modells wurde mittels PLS untersucht, welche Einflussfaktoren dabei die größte Rolle spielen. Anschließend wurde weiter überprüft, inwiefern die Art des genutzten Endgerätes einen Einfluss auf die wahrgenommene Nutzung und Zufriedenheit mit den Lernanwendungen hat.

Konform mit bisherigen Ergebnissen zur Nutzungsintention zeigt sich, dass die Faktoren des erwarteten Mehrwerts und erwarteten Aufwands auch im vorliegenden Fall am wichtigsten beim selbstgesteuerten Mobile Learning sind. Im Falle der Aktivierungsübungen im Vorlesungssaal durch Mobile Learning haben jedoch soziale Einflüsse und unterstützende Bedingungen einen größeren Einfluss. Aus Forschungssicht ist daher festzuhalten, dass weitere Untersuchungen zum Mobile Learning möglicherweise differenzierter vorgehen sollten als bisher. Die Unterschiede zwischen selbstgesteuertem und präsenzgebundenem Mobile Learning sind zumindest im vorliegenden Fall derartig groß, dass die Szenarien explizit unterschieden werden sollten. Aus praktischer Sicht sollten Lehrende, die Mobile Learning einsetzen wollen, sich zunächst über das genaue Einsatzszenario im Klaren sein. Im Falle des selbstgesteuerten Mobile Learnings, zumindest auf Basis von Web Based Trainings und Videos, sollte den Studierenden ein eindeutiger Mehrwert geboten werden. Sie sollten das Gefühl haben, die Nutzung der Lernanwendungen helfe ihnen effektiv bei der Klausurvorbereitung. Zudem sollte die Benutzung des Systems möglichst einfach sein, damit gerade im Falle optionaler Lernmaterialien überhaupt die Bereitschaft besteht, Zeit in die Nutzung zu investieren. Im Falle des Einsatzes von Mobile Learning zur Aktivierung in Präsenzveranstaltungen müssen Dozierende zeigen, dass sie die Beteiligung der Studierenden erwarten. Sie müssen Hilfestellungen geben und den Lernenden ausreichend Zeit 
zur Tätigung ihrer Eingaben einräumen. Der Mehrwert ist weniger zentral, da die Studierenden keine zusätzliche Zeit investieren, sondern sich die Lernanwendungen in den Vorlesungsablauf eingliedern.

In Bezug auf den Zusammenhang zwischen der Art des Endgerätes und der Lernendenzufriedenheit sowie Nutzung der Mobile Learning-Anwendungen finden sich Hinweise, dass die generelle Interaktivität und die einzelnen Aktivierungsübungen in der Vergleichsgruppe der Nutzer von Tablet PCs positiver wahrgenommen werden. Bei den Videos zeichnet sich ein anderes Bild ab. Dort fallen die Ergebnisse in der Gruppe der Laptop-Nutzenden positiver aus, was vermutlich damit einhergeht, dass Multi-Tasking sowie die Nachbearbeitung der Vorlesung mithilfe des Videos auf einem Laptop besser möglich sind.

Die Studie unterliegt verschiedenen Einschränkungen. So erfolgte die Vergabe der Endgeräte nicht zufällig, Studierende konnten sich für ein mobiles Endgerät ihrer Wahl bewerben. Zudem existieren in einem komplexen Umfeld wie einer kompletten Vorlesung vermutlich zahlreiche weitere kleinere oder größere Faktoren, die ebenfalls die Nutzungsintention beeinflussen und hier nicht beachtet wurden. Die Verschiedenheit der Lernanwendungen erschwert zudem den Vergleich der Ergebnisse des selbstgesteuerten und präsenzgebundenen Mobile Learnings untereinander sowie mit den anderen vorgestellten Studien. Dennoch deuten die Ergebnisse auf deutliche Unterschiede zwischen den beiden Anwendungskontexten hin und liefern konkrete Hinweise für Praktiker zu Gestaltung eigener Einsatzszenarien für das Mobile Learning.

\subsection{Literatur}

Benson Soong, M. H., Chuan Chan, H., Chai Chua, B. \& Fong Loh, K. (2001). Critical success factors for on-line course resources. Computers \& Education 36 (2), 101-120.

Chin, W. W. (1998). The Partial Least Squares Approach to Structural Equation Modeling. In G. A. Marcoulides (Hrsg.), Modern Methods for Business Research. London: LEA.

Cohen, P. A. (1981). Student ratings of instruction and student achievement: A meta-analysis of multisection validity studies. Review of Educational Research 51 (3), 281-309.

Crouch, C. \& Mazur, E. (2001). Peer instruction: Ten years of experience and results. American Journal of Physics 69 (9), 970.

Davis, F. D. (1989). Perceived usefulness, perceived ease of use, and user acceptance of information technology. MIS Quarterly 13(3), 319-340. 
Duncan-Howell, J. A. \& Lee, K. T. (2007). M-Learning- Innovations and Initiatives: Finding a place for mobile technologies within tertiary educational settings. Singapore: Ascilite.

El-Gayar, O. F. \& Moran, M. (2006). College Students' Acceptance of Tablet PCs: an Application of the UTAUT Model. DSI Annual Meeting. San Antonio: TX.

Ho, C. T. B. \& Chou, Y. T. T. (2009). The Critical Factors for Applying Podcast in Mobile Language Learning. ICIME.

Motiwalla, L. F. (2007). Mobile learning: A framework and evaluation. Computers \& Education 49 (3), 581-596.

Olmsted III, J. (1999). The mid-lecture break: When less is more. Journal of Chemical Education 76 (4), 525-527.

Sharples, M., Taylor, J. \& Vavoula, G. (2005). Towards a theory of mobile learning. Proceedings of mLearn 2005, 1-9.

Straub, D. \& Burton-Jones, A. (2007). Veni, vidi, vici: Breaking the TAM logjam. Journal of the Association for Information Systems 8 (4), 223-229.

Straub, D., Limayem, M. \& Karahanna-Evaristo, E. (1995). Measuring System Usage: Implications for IS Theory Testing. Management Science 41 (8), 1328-1342.

Sun, P.-C., Tsai, R. J., Finger, G., Chen, Y.-Y. \& Yeh, D. (2008). What drives a successful e-Learning? An empirical investigation of the critical factors influencing learner satisfaction. Computers \& Education 50 (4), 1183-1202.

Thurmond, V. \& Wambach, K. (2004). Understanding interactions in distance education: A review of the literature. International Journal of Instructional Technology and Distance Learning.

Venkatesh, V., Morris, M. G., Davis, G. B. \& Davis, F. D. (2003). User acceptance of information technology: Toward a unified view. MIS Quarterly 27 (3), 425-478.

Wang, Y. S., Wu, M. C. \& Wang, H. Y. (2009). Investigating the determinants and age and gender differences in the acceptance of mobile learning. British Journal of Educational Technology 40 (1), 92-118.

Wegener, R., Bitzer, P., Oeste, S. \& Leimeister, J. M. (2011). Motivation und Herausforderungen für Dozenten bei der Einführung von Mobile Learning. 41. Jahrestagung der Gesellschaft für Informatik. Berlin, Germany. 